\title{
LTE network performance evaluation based on effects of various parameters on the cell range and MAPL
}

\author{
Asaad. S. Daghal ${ }^{1}$, Haider Mohammed Turki Al-Hilfi² \\ ${ }^{1}$ Al-Furat Al-Awsat Technical University, Kufa, Iraq \\ ${ }^{2}$ Directorate General of Vocational Education, Ministry of Education, Iraq
}

\section{Article Info \\ Article history: \\ Received Apr 14, 2021 \\ Revised May 1, 2021 \\ Accepted May 5, 2021}

\section{Keywords:}

eNB

EPC

LTE

MAPL

MCS

MIMO

\begin{abstract}
These days long term evaluation (LTE) is considered the common mobile technology around the world and there is a need to maximize the network performance to satisfy the increased demand in terms of the cell capacity and coverage. These are many parameters in the network configuration and in the surrounded environment, which have great effects on the network performance. Examples of parameters are the system overhead rations, the required capacity of the network, neighbor cell load, and link budget parameters. The determination of the optimum configuration parameters, which achieve the best network performance, is a main step in the planning process in addition to it is continuous step in network optimization phase. In this study, the effects of some parameters will be investigating to get the best parameters that achieve the best network performance in terms of capacity of the cells and coverage area. The study will start by discussing introduction about LTE network components and protocols, and then the main parameters of the protocols will be revising. The study will display the results of changing many parameters related to LTE protocols and surrounding environment parameters on the LTE network performance.
\end{abstract}

This is an open access article under the CC BY-SA license.

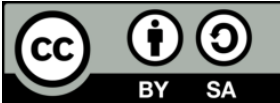

\section{Corresponding Author:}

Asaad. S. Daghal

Department of Avionics Engineering

Engineering Technical College-Najaf

Al-Furat Al-Awsat Technical University, Kufa, Iraq

Email: ad466kent@atu.edu.iq

\section{INTRODUCTION}

4G mobile network is the common technology these days because it has high throughput comparing to $3 \mathrm{G}$ and $2 \mathrm{G}$ mobile networks. $4 \mathrm{G}$ LTE can deliver high mobile data rate that reach $300 \mathrm{Mbps}$ depending on different options like the type of deployed multiple-input and multiple-output (MIMO) that far exceeds the throughput. The number of 4G LTE subscribers reached 1.2 billion in 2016 and estimated to be 4.3 billion by the end of 2021 [1]. Network planning stage is the most vital step in establishment of 4G-LTE mobile network. Planning stage start with network dimensioning which focus on getting the estimated number of radio base-stations (eNodeB) in the network. Two things must be gained to get the estimated number of eNBs, coverage requirements and capacity needs. LTE network has many parameters, which can be optimize to get the maximum network performance, these network parameters must be understood to prevent network performance degradation. link budget $(\mathrm{LiBu})$ is the process of getting the maximum loss allowed in the wireless link. This value used to get the cell range using different clutter types [2], [3].

There are many categories in LTE planning process, one of them is physical cell ID (PCI) planning because LTE requires physical layer cell identity in each cell (maximum 504 PCI organized into 168 groups 
of 3), and there is no need to frequency planning because the frequency reuse factor is one. Additional areas in LTE protocols need to be planned in LTE like physical random access channel (PRACH), physical uplink control channel (PUCCH) and physical downlink control channel ( $\mathrm{PDCCH})$ planning. The remaining parts of this paper is presented as follows: In Section 2, a brief technical overview regarding LTE structure and features is provided. Section 3 presents the planning parameters which used in planning process. Results are listed in part four and conclusion is illustrated in Section 4.

\section{LTE NETWORK STRUCTURE AND PARAMETERS}

LTE network involves two main parts; namely, the radio access and network core parts. The core side is called evolved-packet core (EPC) and the radio part is known evolved-universal terrestrial access network (EUTRAN). The main functions of EPC are to deliver access to other networks based on internet protocol (IP) and provide different functions for idle and active equipment. The functions of EPC are performed using the following nodes, packet-data network gateway (P-GW), serving gateway (S-GW), and mobility-management entity (MME) [4], [5]. LTE utilize OFDMA in downlink and SCFDMA in uplink plus MIMO support. orthogonal frequency-division multiple-access (OFDMA) delivers good spectral efficiency, low latency, and high rates [6]-[8]. The eNB is only element in the access network compared with $2 \mathrm{G}$ and $3 \mathrm{G}$ networks, it executes many functions like dynamic network resource allocation (scheduling), IP header compression/de-compression, handover, and many others. These eNBs is connected with each other by an interface called X2 interface, and connected to EPC using S1 interface. Figure 1 display the basic structure of 4G-LTE network components.

In 2008 release 8 of LTE specifications was ready, some references named it $3.9 \mathrm{G}$ because it didn't satisfy IMT requirements related to maximum data rate. Release 9 was introduced by ending of 2009 with many new features. In 2010, new release was introduced (release 10-LTE-Advanced (LTE-A)) as a huge step in LTE evolution, some references named it $4 \mathrm{G}$ because it is the first system satisfy IMT requirement. These new features involve multiple-input multiple-output (MIMO), carrier aggregation (CA), techniques, coordinated multi-point transmission/reception (CoMP), heterogeneous networks (HetNets), and relaying. The current work is now ongoing for Release 16 that is called 5G phase 2 [9]. LTE in the $5 \mathrm{GHz}$ unlicensed spectrum, namely license assisted access (LAA) and LTE-Unlicensed technologies, is expected to grow in the near future like licensed spectrum due to increasingly loaded. For mobile operators, this offers a solution enabling them to utilize the free unlicensed bands along with a licensed cell using carrier aggregation (CA) [10].

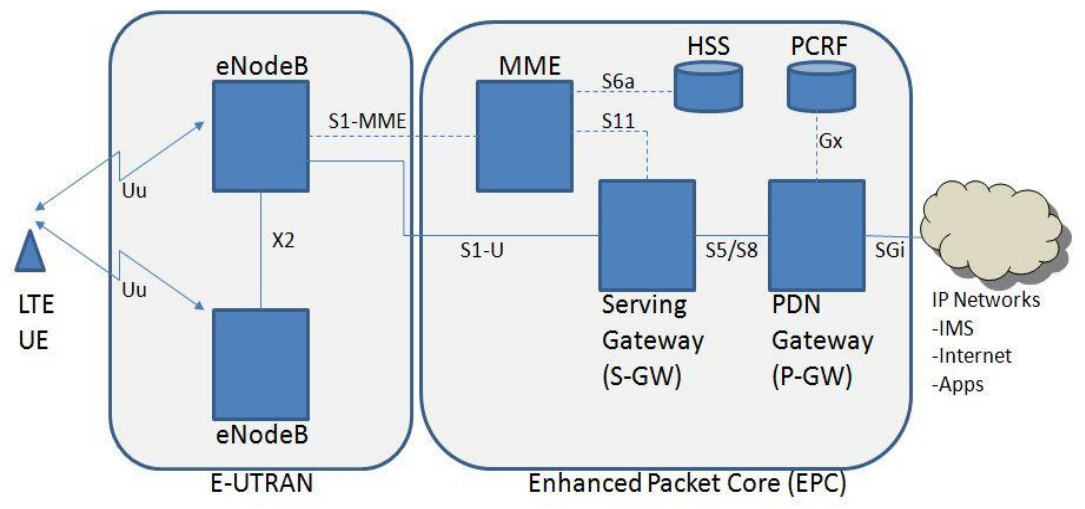

Figure 1. LTE network components

LTE deploy OFDMA, single carrier-frequency division multiple access (FDMA) as multiple access methods specified for LTE downlink (DL), and uplink (UL), respectively. In OFDMA the band are divided into sub-carriers spaced by $15 \mathrm{kHz}$ and 12 adjacent sub-carriers that together occupy $180 \mathrm{kHz}$, form a unit of assigned resources, which named resource block (RB). RB is the minimum bandwidth allocation possible. And from time view these 12 subcarriers has $0.5 \mathrm{~ms}$ which called slot. The network bandwidth divided to a fixed group of RBs as listed in Table 1 [11].

The media access control (MAC) layer in eNB performs scheduling functions, which aim to assign RBs to different service in UL and DL directions. This scheduler makes assignment decisions every transmission time interval (TTI), which has 1 ms duration, by assigning RBs to mobile subscribers. The scheduler also determines different transmission parameters like transmitted power and modulation/coding scheme (MCS), which pointed to as radio link adaptation [12]. Cell reference signals (CRS) is transmitted in 
a predetermined RBs and is used in downlink channel estimation by different users. CRS are used for feedback calculation and demodulation [13]-[15].

\begin{tabular}{cc} 
Table 1. Resource blocks vs BW \\
\cline { 2 - 2 } BW-MHz & RB \\
\hline 1.4 & 6 \\
3 & 15 \\
5 & 25 \\
10 & 50 \\
15 & 75 \\
20 & 100 \\
\hline
\end{tabular}

\section{PLANNING PARAMETERS}

Link budget $(\mathrm{LiBu})$ is a powerful tool, which can be used in different network performance calculations. By using empirical models and theoretical equations and putting them together with configuration tables and load values, the whole network can be simulated [16]. Link budget calculations are implemented in dimensioning stage in the planning process; it involves a sequential step of calculations that have different needs like antenna radiation pattern, simulation capacity requirements, and coverage requirements [17]. Dimensioning process output is the estimated eNB numbers which satisfy the capacity/coverage requirements. The second phase in planning process is the detail planning, which is done by using planning tools for this purpose like U-net or Atoll software [18]. In LiBu, the different losses, gains, and margins are analyzed at receiver and transmitter to give maximum allowable path loss (MAPL) which achieve the needed receiver signal strength. Link budget equations comprise many parameters like [19]: a) transmitted power, b) antenna gain of transmitter/receiver, c) different Losses, d) different margins, and e) receiver sensitivity.

The dimensioning stage starts by getting effective isotropic radiated power (EIRP) by adding the gain of the antenna (GAnt) plus transmitted power (PTX) then subtracts it from different losses. EIRP is the product of transmitter power and transmitting antenna gains w.r.t isotropic antenna of a radio transmitter.

$$
E I R P=P T X+G A n t+G M I M O-L \text { feeder }-L \text { body }-I M
$$

Where GMIMO is the gain due to transmit diversity techniques, L feeder is the losses in the feeder, L body is the loss due to adherence of the transceiver to the body, IM is the interference margin, which depends on the load of the neighbor cells.

The next step is to get receiver sensitivity, which is the minimum amplitude of required input signal to get a specific signal with a specific SINR, or other specified criteria:

$$
S R X=-174 \mathrm{dBm} / \mathrm{Hz}+10 \log (15 \mathrm{KHz} * 12 \mathrm{RB})+\mathrm{NF}+\operatorname{SINR}
$$

Where $174 \mathrm{dBm} / \mathrm{Hz}$ is the thermal noise, SINR is the signal strength to interference power plus noise power ratio, and $\mathrm{RB}$ is the resource block numbers. NF is the device noise figure, which depends on the hardware design of receiver equipment. NF represents an additive noise that is generated by various hardware components. The main output is MAPL, which is obtained by subtracting EIRP from SRX and is used to get cell range and consequently inter-site distance (ISD) after considering radio propagation issues (clutters). The evaluation was done using COST HATA-231 model, this model is used for 4G-LTE in different environments [20]-[23]. Radio propagation environments (clutters). Include dense urban, sub-urban, urban, rural environments. The difference between them in the existence of buildings, trees, hills, and people within these environments that affect the propagation of signals [24]. Table 2 lists the dimensioning parameters that used in the estimation of the results.

Table 2. LTE dimensioning parameters

\begin{tabular}{cccccccc}
\hline $\begin{array}{c}\text { Operating } \\
\text { frequency }\end{array}$ & BW & Downlink/Uplink rate & eNB power & Antenna gain & Noise figure (DL/UL) & BLER & Default load \\
\hline $2600 \mathrm{MHz}$ & $10 \mathrm{MHz}$ & $4096 / 384 \mathrm{~Kb} / \mathrm{s}$ & $20 \mathrm{~W}(43 \mathrm{dBm})$ & $18 \mathrm{dBi}$ & $7 \mathrm{~dB} / 2.2 \mathrm{~dB}$ & $10 \%$ & $50 \%$ \\
\hline
\end{tabular}

\section{RESULTS AND DISCUSSION}

To get the maximum cell range in LTE network, the required cell-edge throughput must be determined which is used in getting required signal-to-interference -noise ratio (SINR). In (1) and (2) are 
used to get MAPL, which considered an input to clutter equations. Table 3 lists the cell ranges with the required maximum cell edge throughput. As the table, shows when the required throughput increases the cell range must be decrease to obtain the required throughput. This is because as the required throughput increases the MCS order must be increase, which only can be implemented close to the cell site. Figure 2 displays the relation between the use of different MCS and MAPL, the figure shows that MAPL decreases with increasing MCS index, which means decreasing the cell range. In the same context the percentage of used RB is decreased which mean increasing the cell capacity. So, there is tradeoff between the capacity and the coverage when increasing MCS index. Figure 3 displays the relation between using different MCS and the resultant cell range. The figure shows reduction of cell range with increasing of MCS index. This means that if the network is planned to use high order MCS, the number of eNBs that used to cover the network is increased due to reduction in cell ranges. Note that the blue line represents the cell range that resulted from MAPL calculation using urban clutter and orange line represent cell rage without considering clutters. The difference between them related to the clutter's calculations take other losses and propagation issues in to considerations.

Table 3. Cell range in different required throughput

\begin{tabular}{cc}
\hline Throughput $(\mathrm{kb} / \mathrm{s})$ & Cell range-km \\
\hline 512 & 0.73 \\
1024 & 0.68 \\
2048 & 0.56 \\
4096 & 0.41 \\
8192 & 0.16 \\
\hline
\end{tabular}

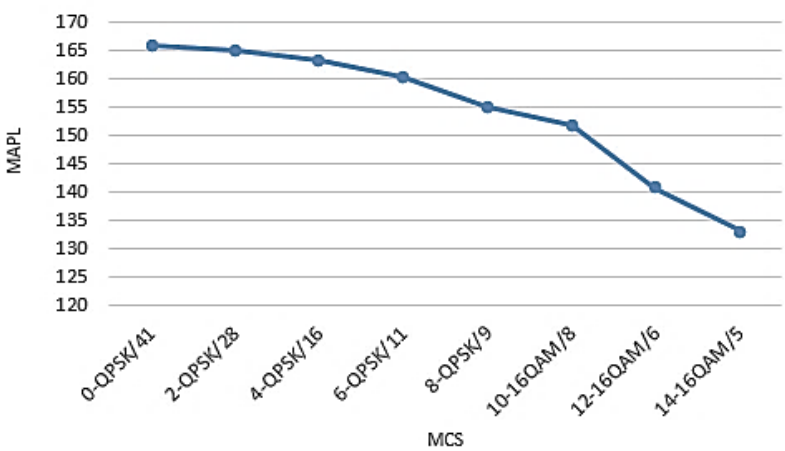

Figure 2. MAPL vs MCS

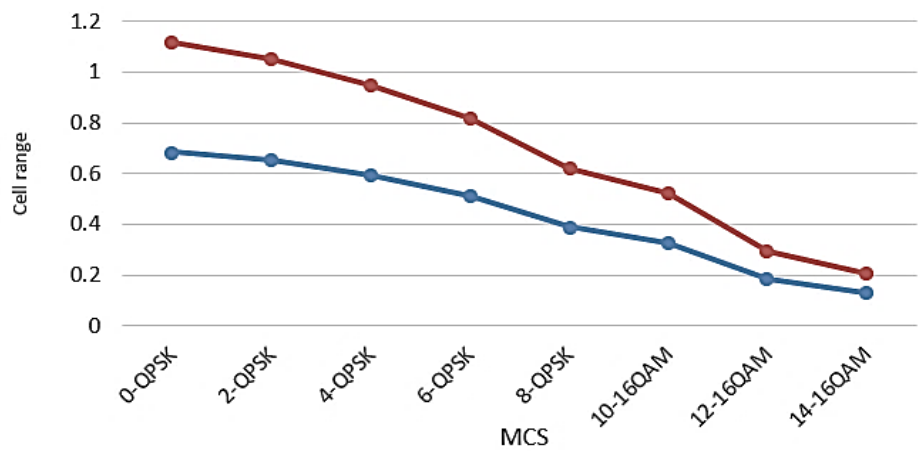

Figure 3. Cell range vs MCS

RB utilization vs MCS index is shown in Figure 4. The figure declares that the RB usage percentage is decreased with using less robust MCS (high order MCS). The percentage decreased from $86 \%$ using 6QPSK to $13 \%$ using 28-64QAM. Using less RB to meet the required throughput increase the cell capacity in terms of the active number of subscribers in the same time. It is assumed that the required cell-edge throughput is $4069 \mathrm{~kb} / \mathrm{s}$, so the number of active users is increased by factor of 7 , but the cell range is decreased as shown in Figure 3. 


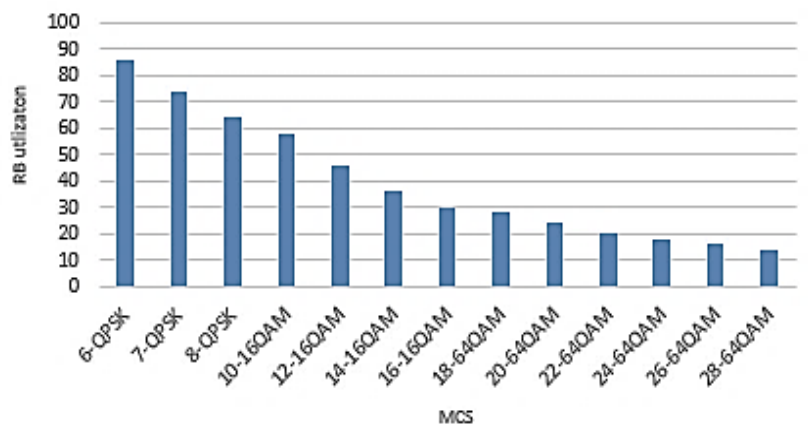

Figure 4. RB utilization vs MCS

Figure 5 displays the relation between the MCS efficiency and MCS scheme, as shown in the figure the modulation efficiency which is measured in bit per modulated symbol increases with MCS order, this is due to decreasing redundancy bits in high order modulation and increasing the bit per symbol. Relating to TS 36.213 from 3GPP, to deploy the different MCS in LTE receivers, the signal must meet the required SINR that shown in Figure 6. The figure declares that to utilize 28-64QAM the SINR must be 21.68 dbm and to achieve it we must be very close to eNB site. Figure 7 illustrates the relation between neighbor load percentage (percentage of occupied RBs) and interference margin that is considered when calculate MAPL.

The Figure 7 shows that with increasing the load percentage the interference level on the target cell increases, so the interference margin that included in MAPL calculation is increased. The values of interference margin are calculated using (3).

$$
I M=-10 \log [1-\operatorname{SINR} * \eta / G]
$$

Where $\eta$ is neighbor cell load, $G$ is geometry factor which is signal to interference at cell edge which is depend on network geometry (cell area location probability) and antenna configuration, $\mathrm{G}=-0.03$ from NSN system level simulation for 3GPP macro case [25]. Figure 8 shows the effect of load percentage on MAPL without considering clutter. Above $50 \%$ load, the reduction of MAPL becomes very large which decrease the cell range.

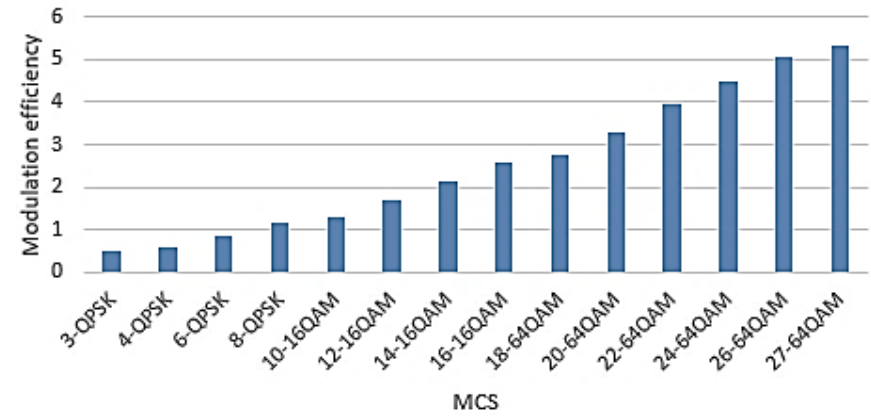

Figure 5. MCS order vs modulation efficiency

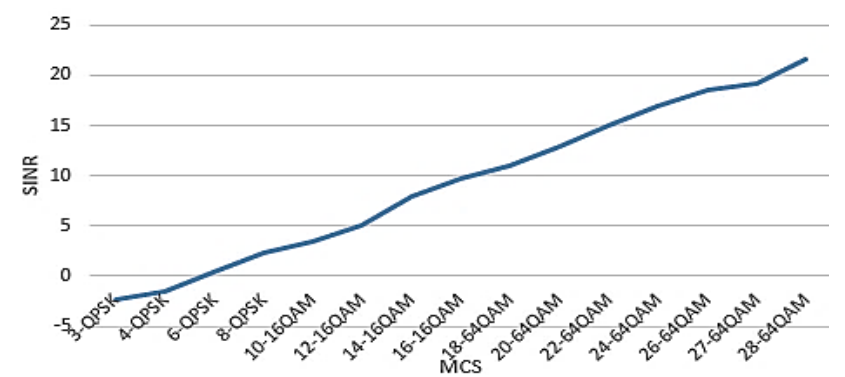

Figure 6. SINR vs MCS 


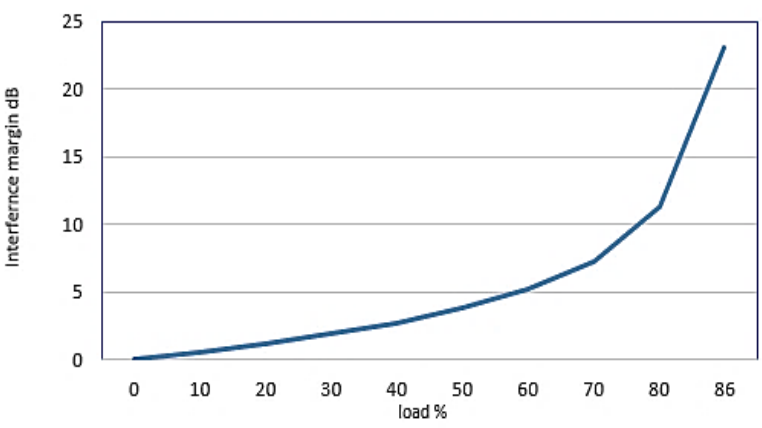

Figure 7. Load \% vs interference margin

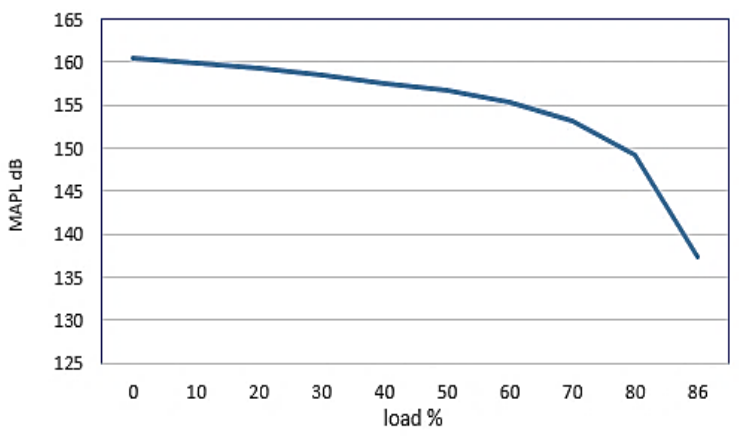

Figure. 8 Cell load against MAPL

The control channels overhead in DL and UL directions affect the capacity of eNB cells. Table 4 declares the relation between the PDCCH symbols and total percentage of control RBs. It shows that using 3 symbols for PDCCH consume $20 \%$ from the total RBs. So, we have to minimize the amount of control messages to decrease the needed number of PDCCH symbol, the best solution is to make the number of PDCCH symbol dynamic not fixed values. Table 5 declare the relation between Number of PRBs for PUCCH and the total system overhead.

Table 4. PDCCH symbol vs used RB \%

\begin{tabular}{cc}
\hline PDCCH symbol & RB \% \\
\hline 1 & 10 \\
2 & 12 \\
3 & 20 \\
4 & 27 \\
\hline
\end{tabular}

Table 5. Total system overhead vs PUCCH RBs

\begin{tabular}{cc}
\hline Number of RBs for PUCCH & Total System Overhead \% \\
\hline 1 & 19 \\
2 & 20 \\
3 & 22 \\
4 & 24 \\
5 & 26 \\
\hline
\end{tabular}

\section{CONCLUSION}

The choice of the planning parameters in LTE network planning processes has great effect on the network performance from coverage, and capacity perspectives. The paper presents the effects of different control channels like PDCCH/PUCCH on the used RBs and total system overhead. The effect of load percentage of the neighbor cells on MAPL has been investigated, the results show that above 50\% the MAPL is decreased which leads to small coverage area. The study declares that using high order, MCS leads to high throughput but in same time it decreases the cell range, so there is trade-off between the capacity of eNB cells and coverage area in LTE network design. From the study it is clear that we have to choose the location of eNBs close to the users as much as possible, this is to decrease the utilization of RBs to allow high throughput and eNB capacity

\section{REFERENCES}

[1] Tchao, E. T., Gadze, J. D., and Agyapong, J. O., "Performance Evaluation of a Deployed 4G LTE Network," International Journal of Advanced Computer Science and Applications (IJACSA), vol. 9, no. 3, pp. 165-178, 2018, doi: 10.14569/IJACSA.2018.090325.

[2] Jaber, M., Dawy, Z., Akl, N., and Yaacoub, E., "Tutorial on LTE/LTE-A cellular network dimensioning using iterative statistical analysis," IEEE Communications Surveys \& Tutorials, vol. 18, no. 2, pp. 1355-1383, 2015, doi: 10.1109/COMST.2015.2513440.

[3] Jha, S. K., Rokaya, R., Bhagat, A., Khan, A. R., \& Aryal, L., "LTE Network: Coverage and Capacity Planning-4G Cellular Network Planning around Banepa," 2017 International Conference on Networking and Network Applications (NaNA), pp. 180-185, 2017, doi: 10.1109/NaNA.2017.23.

[4] Al-Hilfi, H. M. T., and Daghal, A. S., "Effects of Varying LTE Link Budget Parameters on MAPL and Cell Range," Journal of Communications, vol. 15, no. 7, 2020, doi: 10.12720/jcm.15.7.583-587.

[5] Ali Jemmali, "Performance evaluation and analysis of mimo schemes in lte networks environment," Ph. D. dissertation, École Polytechnique de Montréa, 2013.

[6] Abed, G. A., Ismail, M., and Jumari, K., "Modeling and Performance Evaluation of LTE Networks with Different TCP Variants," International Journal of Electrical, Computer, Energetic, Electronic and Communication Engineering, vol. 5, no. 3, pp. 443-448, 2011. 
[7] Hamamreh, J. M., Hajar, A., and Abewa, M., "Orthogonal frequency division multiplexing with subcarrier power modulation for doubling the spectral efficiency of 6G and beyond networks," Transactions on Emerging Telecommunications Technologies, vol. 31, no. 4, 2020, doi: 10.1002/ett.3921.

[8] Le, Nam-Tran, Dhammika Jayalath, and Jacob Coetzee., "Spectral-efficient resource allocation for mixed services in OFDMA-based 5G heterogeneous networks," Transactions on Emerging Telecommunications Technologies, vol. 29, no. 1, 2018, doi: 10.1002/ett.3267.

[9] Joud, M., "Pico Cell Range Expansion toward LTE-Advanced Wireless Heterogeneous Networks," Master's thesis, Universitat Politècnica de Catalunya (UPC), 2013.

[10] Saha, S. K., Vlachou, C., Koutsonikolas, D., and Kim, K. H., "DeMiLTE: Detecting and Mitigating LTE Interference for Enterprise Wi-Fi in $5 \mathrm{GHz}$," Proceedings of the Twentieth ACM International Symposium on Mobile Ad Hoc Networking and Computing, 2019, pp. 31-40, doi: 10.1145/3323679.3326501.

[11] Pires, R., "Coverage and Efficiency Performance Evaluation of LTE in Urban Scenarios," Diss. M. Sc. thesis, Instituto Superior Técnico, Lisbon, Portugal, 2012.

[12] Iosif, O., and Bănică, I., "LTE Performance Evaluation Based on two Scheduling Models," International Journal on Advances in Networks and Services, vol. 5 no. 1 \&, 2, pp. 58-68, 2012.

[13] Meidlinger, M., and Wang, Q., "Performance Evaluation of LTE Advanced Downlink Channel Estimators," International Conference on Systems, Signals and Image Processing (IWSSIP), 2012, pp. 252-255.

[14] Davydov A., Morozov G., "Enhanced Interference Cancellation of Cell-Specific Reference Signals for LTE-A," 2015 IEEE 82nd Vehicular Technology Conference (VTC2015-Fall), 2015, pp. 1-5, doi: 10.1109/VTCFall.2015.7390925.

[15] Kurras, M., Thiele, L., Haustein, T., Lei, W., and Yan, C., "Full dimension MIMO for frequency division duplex under signaling and feedback constraints," 2016 24th European Signal Processing Conference (EUSIPCO), pp. 1985-1989, 2016, doi: 10.1109/EUSIPCO.2016.7760596.

[16] Davidović, M., Tomić, I., Drajić, D., and Čiča, Z., "On the Impact of NB-IoT on LTE MBB Downlink Performance", Telfor Journal, vol. 11, no. 1, pp. 20-24, 2019, doi: 10.1109/TELFOR.2018.8611908.

[17] Bechta K., Rybakowski M., Hsieh F., and Chizhik D, "Modeling of radio link budget with beamforming antennas for evaluation of 5G systems," 2018 IEEE 5G World Forum (5GWF), 2018, pp. 427-432, doi: 10.1109/5GWF.2018.8516969.

[18] Atoll network planning simulation tool, [Online]. Available: http://www.forsk.com/atoll-overview

[19] Sharma, P. K., Sharma, D., and Gupta, A. "Cell Coverage Area and Link Budget Calculations in LTE System", Indian Journal of Science and Technology, vol. 9, no. S1, pp. 1-7, Dec. 2016, doi: 10.17485/ijst/2016/v9iS1/107935.

[20] Talukder, Z. H., Islam, S. S., Mahjabeen, D., Ahmed, A., Rafique, S., and Rashid, M. A., "Cell coverage evaluation for LTE and WiMAX in wireless communication system," World Applied Sciences Journal, vol. 22, no. 10, pp. 1486-1491, 2013, doi: 10.5829/idosi.wasj.2013.22.10.2006.

[21] Saeed, A., Rehman, H. U., and Masood, M. H., "Performance Analysis and Comparison of Radio Propagation Models for Outdoor Environment in 4G LTE Network," 2013.

[22] Akhpashev, R. V., and Andreev, A. V., "COST 231 Hata adaptation model for urban conditions in LTE networks," 2016 17th International Conference of Young Specialists on Micro/Nanotechnologies and Electron Devices (EDM), 2016, doi: 10.1109/EDM.2016.7538693.

[23] Abbas, M. J., "Interoperability Framework for Wireless Standards-Performance Analysis," 2018 Recent Advances on Engineering, Technology and Computational Sciences (RAETCS), pp. 1-5, 2018, doi: 10.1109/RAETCS.2018.8443818.

[24] Kebede, G. M., and Paul, O. O., "Performance Evaluation of LTE Downlink with MIMO Techniques," School of Engineering, Blekinge Institute of Technology, 2010.

[25] Nokia Siemens Network, “LTE RPESS, LTE Link Budget,” pp. 3.1, 2011. 